\title{
Expert System Driven Fuzzy Control Application to Power Reactors
}

\author{
L. H. Tsoukalas, R. C. Berkan, B. R. Upadhyaya and R. E. Uhrig \\ The University of Tennessee \\ Nuclear Engineering Department \\ CONF-9011312--1 \\ DE93 002121 \\ Knoxville, TN 37996-2300
}

\begin{abstract}
For the purpose of nonlinear control and uncertainty/imprecision handling, fuzzy controllers have recently reached acclaim and increasing commercial application. The fuzzy control algorithms often require a "supervisory" routine that provides necessary heuristics for interface, adaptation, mode selection and other implementation issues. Performance characteristics of an on-line fuzzy controller depend strictly on the ability of such supervisory routines to manipulate the fuzzy control algorithm and enhance its control capabilities.

This paper describes an expert system driven fuzzy control design application to nuclear reactor control, for the automated start-up control of the Experimental Breeder Reactor-II. The methodology is verified through computer simulations using a valid nonlinear model. The necessary heuristic decisions are identified that are vitally important for the implemention of fuzzy control in the actual plant. An expert system structure incorporating the necessary supervisory routines is discussed. The discussion also includes the possibility of synthesizing the fuzzy, exact and combined reasoning to include both inexact concepts, uncertainty and fuzziness, within the same environment.
\end{abstract}

\section{Introduction}

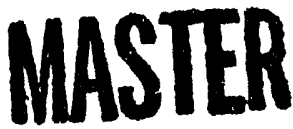

Standard logic rules used in conventional control systems represent a model of the operational rules in which the information describing the nature of the process

$$
\begin{aligned}
& 1 \text { aISTRIBUTION OF THIS DOCOMENT IS UNLIMTE } \\
& \text { FCO T-88ER/2824 }
\end{aligned}
$$


is confined to the exact definitions of an event/mode. In reality, system operations often deviate from prescribed definitions and set-points. Most of the control problems arise due to the severness of these mismatches; their solutions require an appropriate interpretation of the inexactness. The emergence of fuzzy logic and fuzzy control concepts have brought a new dimension to these problems and generally to the uncertainty management problem. Especially in industrial applications where a system's performance depends on the operator's ability to handle inexactness, the fuzzy algorithms show a potential to enhance the operator's performance and reduce operating risk.

This paper focuses on a fuzzy control application to nuclear reactors. Particular emphasis is given to the automated start-up control of the Experimentai Breeder Reactor-II (EBR-II). The EBR-II operations constitute a good example of operatordriven systems where the inexactness is entirely interpreted using human expertise. A fuzzy control algorithm is developed and implemented in the feedback-loop using a validated nonlinear model. The feedback controller is designed to maintain the plant states within the desired start-up trajectories for a given change in the set points. The set-points of the two control inputs are obtained from actual startup data of the EBR-II. Thus, the plant model is driven by the combination of predetermined control trajectories and a fuzzy control correction.

The implementation of the fuzzy control paradigm within the actual plant environment requires additional considerations. Most importantly the adaptation of the fuzzy control rules for a possible unexpected plant behavior is often necessary to provide robustness. The EBR-II start-up procedure includes steady-state intervals between each $10 \mathrm{MW}$ startup transient. This requires a sequential change in the mode of the transient. Accordingly, the flexibility of the controller needs to be adjusted for the different modes of the overall transient. Another consideration is related to the control system synthesis. The regulation task may be incorporated with the anticipatory paradigm in which the on-line decision making utilizes extended knowledge (anticipated future behavion) as well as on-line information from the plant [4]. This paper introduces the concept of using an expert system as a supervisory routine which manipulates the fuzzy controller to enhance performance

\section{Fuzzy Control Paradigm}

The central notion in fuzzy logic is that of a linguistic variable [1] whose values are words or sentences in a synthetic language. A linguistic variable includes an adjective-like term (and its antonym), a modifier and a connective. The modifier is a measure of intensity which is associated by a possibility distribution. This is often referred to as the membership function in the literature. The fuzzy control policy is represented as a finite collection of rules, called fuzzy productions (FP), of the form

$$
R^{i}: \text { if }\left(X_{1} \text { is } A_{1}^{i}\right) \text { and } \cdots\left(X_{n} \text { is } A_{n}^{i}\right)
$$


then

$$
Y^{i}=a_{0}^{i}+a_{1}^{i} X_{1}+\cdots+a_{n}^{i} X_{n}
$$

where $R^{i}$ is the $i$ th rule; $A_{j}^{i}$ is a linguistic value of $X$, in $R^{i} ; Y^{i}$ is the control variable, and $a_{n}^{i}$ are adjustable parameters. The truth value of the antecedent of $R^{i}$ is given as (minimum is denoted by $\Lambda$ )

$$
W^{i}=A_{1}^{i}\left(X_{1}\right) \wedge \cdots \wedge A_{n}^{i}\left(X_{n}\right)
$$

where $A_{j}^{i}\left(X_{j}\right)$ is the grade of membership of $X_{j}$ in $A_{j}^{i}$. The aggregated value of control $Y$ is a normalized linear combination given by

$$
Y=\frac{W_{1} Y^{1}+\cdots+W_{n} Y^{n}}{W_{1}+\cdots+W_{n}}
$$

The equation above suggests a weighted vote in the decision of control. Generally in a fuzzy production system, all rules are considered to be fired - with different strengths. Of course, rules that fire strongly contribute significantly to the final conclusions.

Fuzzy logic applications to control dynamic systems require fuzzy rules to depend on the past performance of the process more than the batch type operations where the fuzzy rules represent the inexactness among the standard logical propositions. The fuzzy control paradigm for the dynamic, on-line systems includes a table of fuzzy rules (truth table) that relates the state variables to the control variables. The truth table is constructed using a set of previously recorded data during the particular mode of operation. Every line in the truth table corresponds to a unique relationship among the state and control variables of the system. The implementation of control is determined by an interpolative method when the input to the control system does not match with the canonical structure of the table (fuzzy predicate). The linear interpolation also corresponds to the triangle membership function. Note that the linear interpolation between any two points in the phase-plane does not necessarily indicate that the method employs a linear conirol law.

The control law cain be formulated as follows. Consider a process identified by state variables $\left(X_{1}, \cdots, X_{n}\right)$ and control variables $\left(Y^{1}, \cdots, Y^{n}\right)$. Each control variable is calculated by

$$
Y^{i}=\frac{W_{1 i} U_{1}^{i}+\cdots+W_{n i} U_{n}^{i}}{W_{1 i}+\cdots+W_{n i}}
$$

where

$$
U_{j}^{i}=\mathcal{F}\left(X_{j}\right)
$$

and $W_{j i}$ s are trainable weights. The partial controls $U_{j}^{i}$ represent one relationship between the state variable $X_{j}$ and the control $Y^{i}$. The resultant control which 
incorporates the relationships between all of the state variables and $Y^{\circ}$ is the voted average of all contributions.

One important aspect of this approach is the degree of consistency between the measured states and the corresponding elements of the truth table. A sparse distribution of measured states may not easily be interpretable by the table. This brings the robustness issue into the picture. Regardless of the reason of sparseness (unanticipated transients on measurement degradation), the con ${ }^{\prime}$, , system is always desired to inherit some degree of robustness. One way of enhancing the robustness is to identify the characteristic state variable of the system which is the best candidate to represent the overall state of operation. Unfortunately, this is a system related property and the best candidate may not be reliable in many systems. In nuclear reactors however, neutron population can be considered as the characteristic state variable which is a good indicator of the overall performance. The robustness is then enhanced by referring to the reactor power measurement ard identifying the remaining membership functions accordingly.

\section{Fuzzy Controller for EBR-II Plant Start-up}

EBR-II is a $62.5 \mathrm{Mw}(\mathrm{th})$ liquid-metal reactor built for irradiation experiments. The reactor start-up is mainly controlled by plant operators who make on-line judgements about the reactor performance. The operators adjust the control rod position and the intermediate heat ecchanger sodium flow rate by comparing the measured system variables with the reference trajectories or predetermined set points. In this work, the operator is replaced by a fuzzy controller.

The automation of the EBR-II start-up procedure requires a trajectory following strategy for both rod reactivity and secondary scdium flow. Unlike the steady state control of dynamic systems, the trajectory following control imposes some difficulties when incorporated within the fuzzy control paradigm. A new approach that employs the fuzzy control in the feedback loop is tested. The fuzzy block includes a truth table identifying the state and control variables in a common phase plane. The table is constructed using a data set previously recorded during a successful startup at EBR-II. In the feedback loop, the fuzzy block provides a correction to the startup commands (fixed trajectories) ensuring that the plant states remain within the phase plane as represented in the table.

The fuzzy controller is tested through simulations using a previously validated nonlinear model (10 state variables) [2]. The simulation results show that the performance of the control system with the fuzzy block in the feedback loop is comparable to the actual plant control. The rod reactivity and secondary sodium flow trajectories are followed accurately as well as the other state variables of the plant.

The simulations are performed using the model building capabilities of software system MATRIX-x [3]. Figure 1 shows the SYSTEM BUILD [3] blocks representing 
the EBR-1I model, fuzzy controller and the control logic block named "wire". The interior of the fuzzy block is shown in Fig. 2 where the input channels 1.2 and 3 correspond to the on-line readings of reactor power, core exit temperature and intermediate heat exchanger outlet temperature. The output of fuzzy blocks are equally weighted to yield the final form of the control signals (rof=rod position, $\mathrm{wf}=$ flow rate). Figures 3,4 and 5 compare the actual plant data (operator driven) with the simulation results where operators were replaced with the fuzzy controller. The control signals are compared in a similar manner as shown in Figs. 6 and 7.

\section{Expert Systems and Fuzzy Control}

The point of departure in the present research is that the intuitive control strategies employed by the EBR-II operators can be viewed as fuzzy algorithms and/or heuristics. The heuristics are encoded in the knowledge base of an expert system employing the structure of fuzzy productions. In this manner, qualitative information is handled in a rigorous way. Facts are encoded as propositions having the canonical form " $X$ is $A$ ", as shown in the left hand side (LHS) of Eq. 1. In this representation, each constituent clause of the LHS of a fuzzy production is viewed as a constraint on a variable, such as $X_{1}$.

Although the system describes the operator's control strategy based on linguistic statements (in the manner of Eq. 1), there is no general method for calculating, the membership functions for a particular parameter (control, state). The values of the coefficients $a_{j}^{i}$ in Eq. 1 (and hence the weights $W^{i}$ ) are determined through training. Future work will focus on the training aspect. The possibility of using a neural network for this purpose is currently under investigation. In such a case, the expert system functions as the supervisor which coordinates on-line training when necessary. Training, however, can not guarantee that the fuzzy control algorithm will always converge to the correct values of the coefficients. The resulting ambiguity and subjectivity in the implementation of fuzzy control laws are compensated by a modular expert system design, that allows for expansion and modification on the face of experience.

In addition to training functions, the expert system provides the general identification of the system and makes decision about changing the mode of the controller. There are two types of controller modes: normal modes and non-routine modes. The normal modes are: start-up, cold standby, restart, steady power, plant shutdown, and fuel handling. The non-routine modes are: manual or automatic scram, anticipatory shutdown, plant cooldown, and plant heatup.

\section{DISCLAIMER}

This report was prepared as an account of work sponsored by an agency of the United States Government. Neither the United States Gnvernment nor any agency thereof, nor any of their employees, makes any warranty, express or implied, or assumes any legal liability or responsibility for the accuracy, completeness, or usefulness of any information, apparatus, product, or process disclosed, or represents that its use would not infringe privately owned rights. Reference herein to any specific commercial product, process, or service by trade name, trademark, manufacturer, or otherwise does not necessarily constitute or imply its endorsement, recommendation, or favoring by the United States Government or any agency thereof. The views and opinions of authors expressed herein do not necessarily state or reflect those of the United States Government or any agency thereof. 


\section{Conclusions}

A control system using fuzzy productions and applications to the automated startup of the Experimental Breeder Reactor-II has been presented. An expert system supervises the fuzzy controller. The overall system emulates the actions of a plant operator. The operator control strategy is based on linguistic statements which translate into fuzzy productions describing heuristic control rules. A comparison with actual plant data shows that the fuzzy controller performs very well.

In the case of a startup mode, the relation between the state and control variables is often qualitatively known. Fuzzy logic rules are an appropriate way for implementing such an approximate and yet quite robust control strategy. The possibilities of using neural networks for training and employing anticipatory control will be investigated in the future.

\section{References}

[1] L. A. Zadeh, "Fuzzy Algorithms," Information and Control, Vol. 12, pp 94-102, 1968.

[2] R. C. Berkan, B. R. Upadhyaya and R. A. Kisner, "Low-order Modeling of Experimental Breeder Reactor-II", ORNL-TM 6590, 1990.

[3] MATRIX-x WS User's Guide, Integrated Systems, Inc. Version 3.0, 1990.

[4] L. Tsoukalas and J. Reyes-Jimenez, "A Hybrid Expert System-Neural Network Methodology for Anticipatory Control in a Process Environment," in Proc. of The Third International Conference on Industrial and Engineering Applications of Artificial Intelligence and Expert Systems IEA/AIE-90, ACM/SIGART, Charleston SC, July 15-18, 1990. 


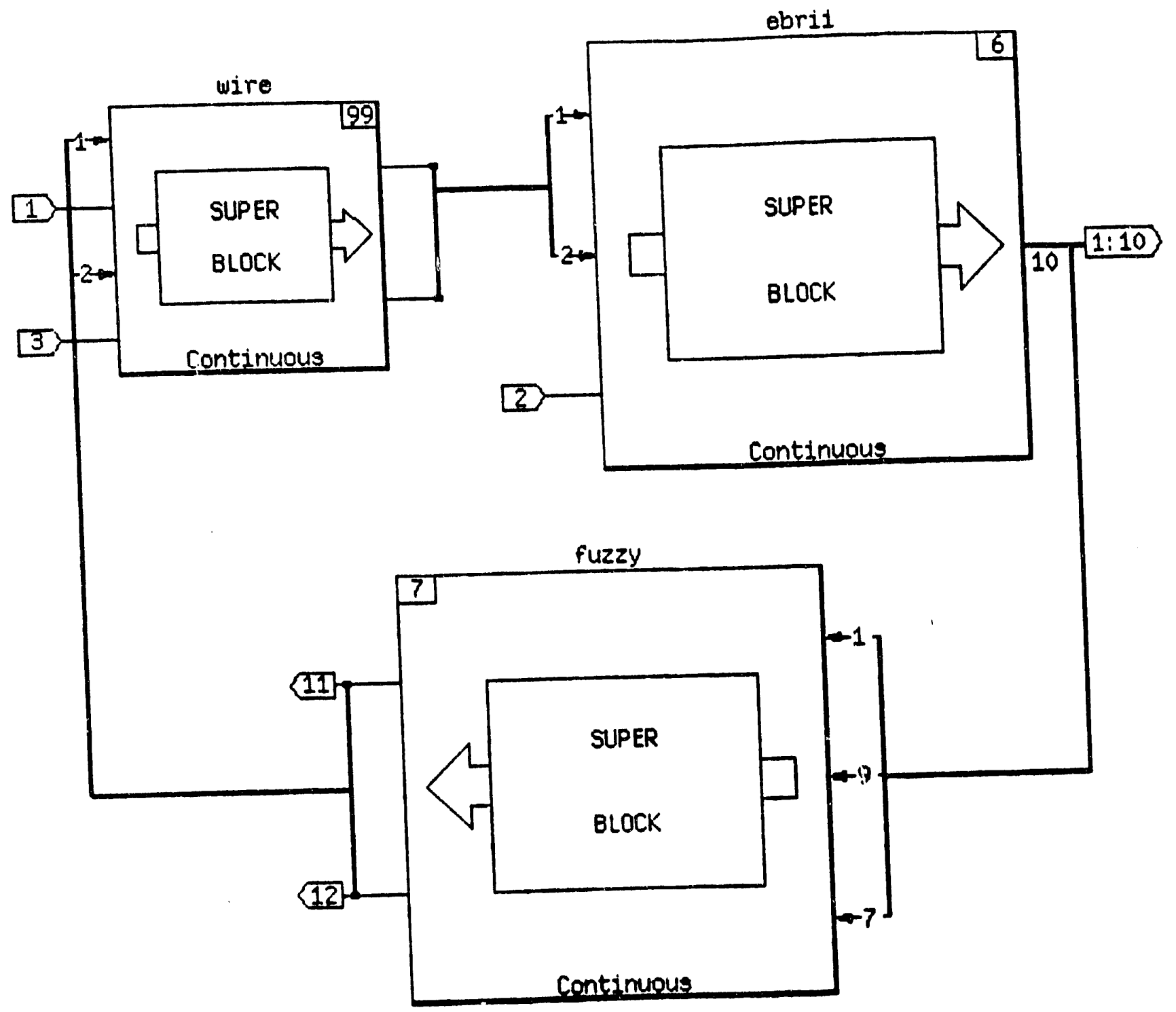

Figure 1. Three super blocks showing the EBR-II, fuzzy
controller and the control strategy implementation. 

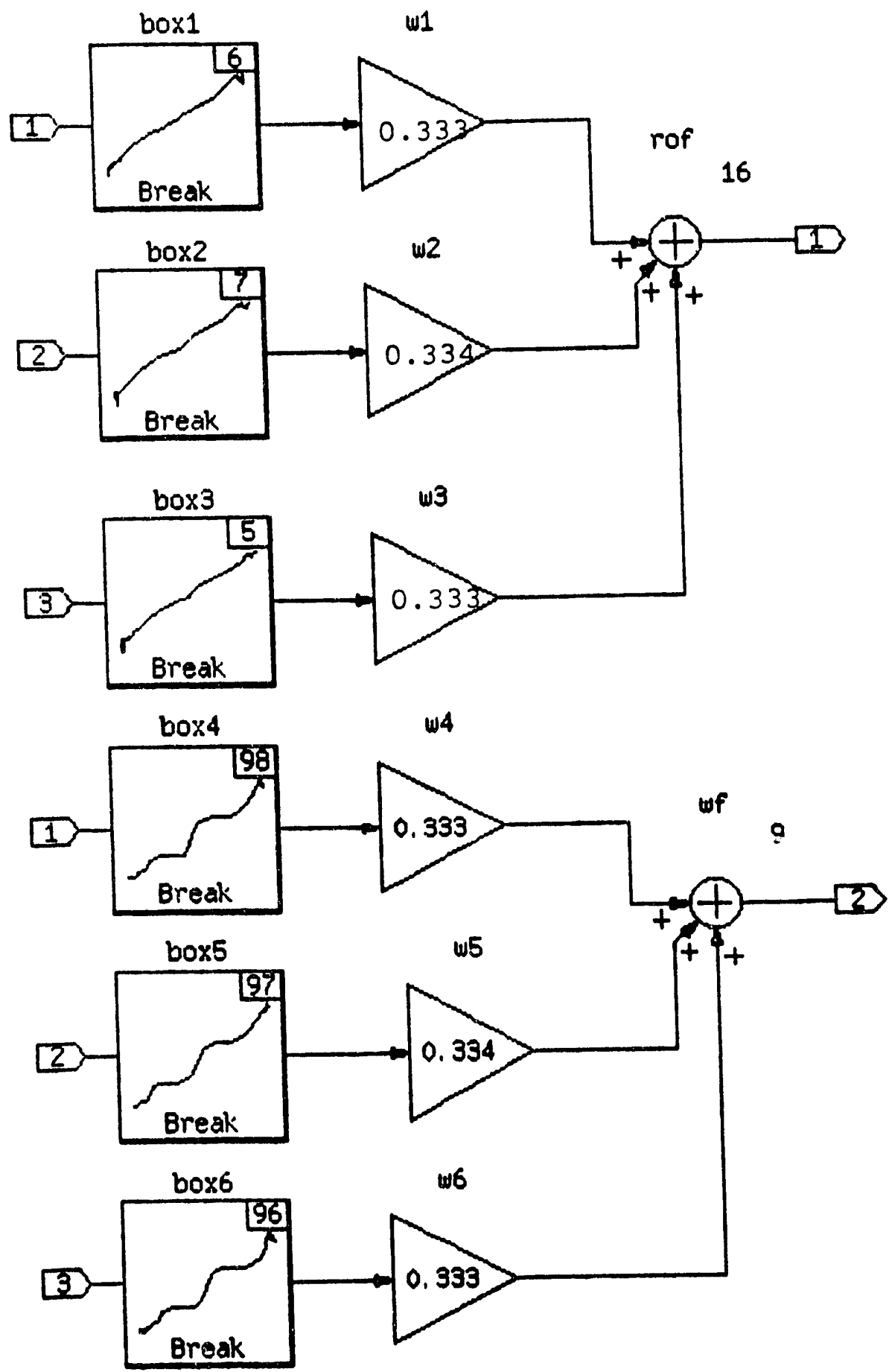

Figure 2. Details of the fuzzy controller showing
individual control law generation using phase plots of process variable vs. control input. 


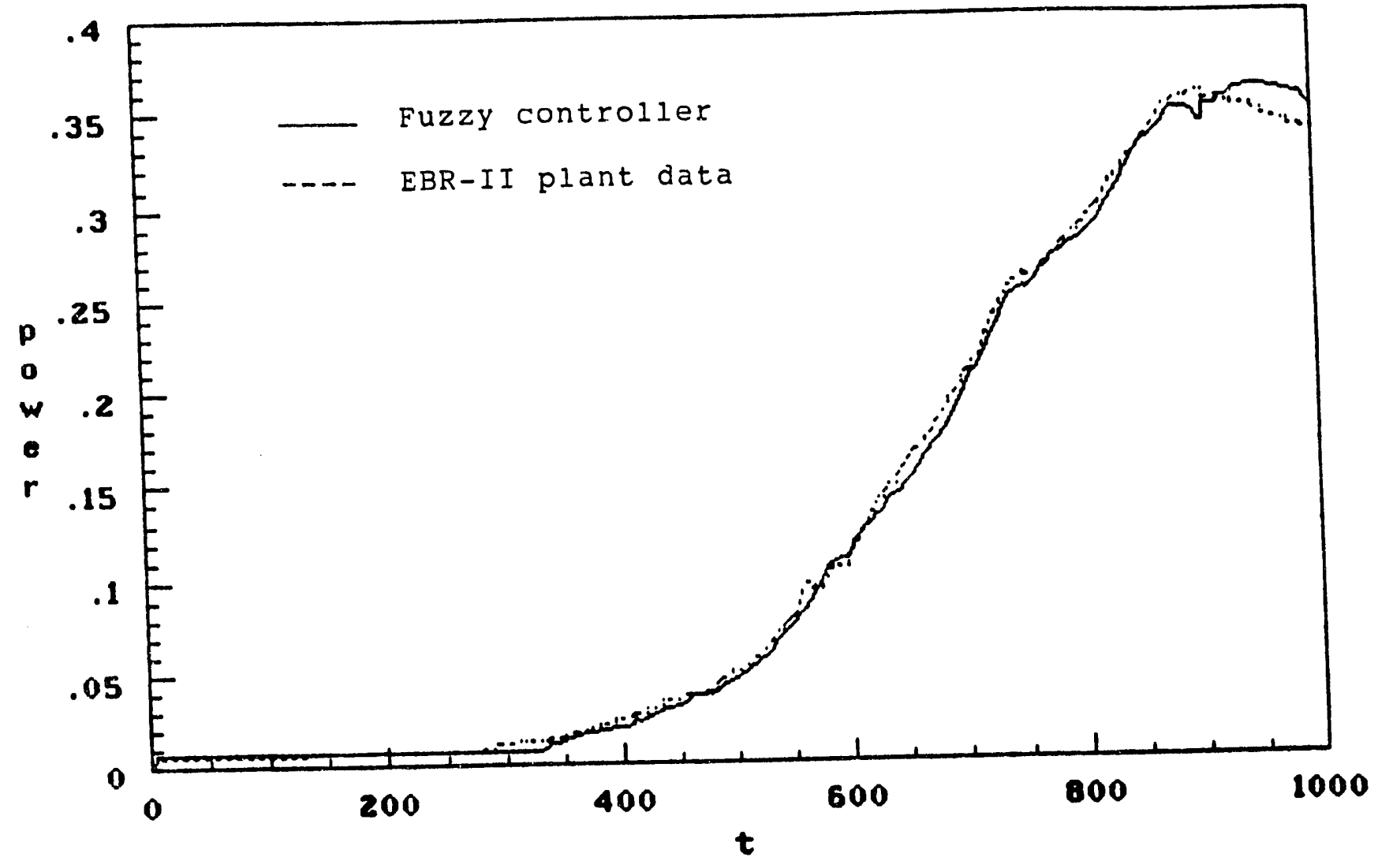
Figure 3. Comparison of reactor power (\%) response due to
the fuzzy controller and EBR-II plant data. 


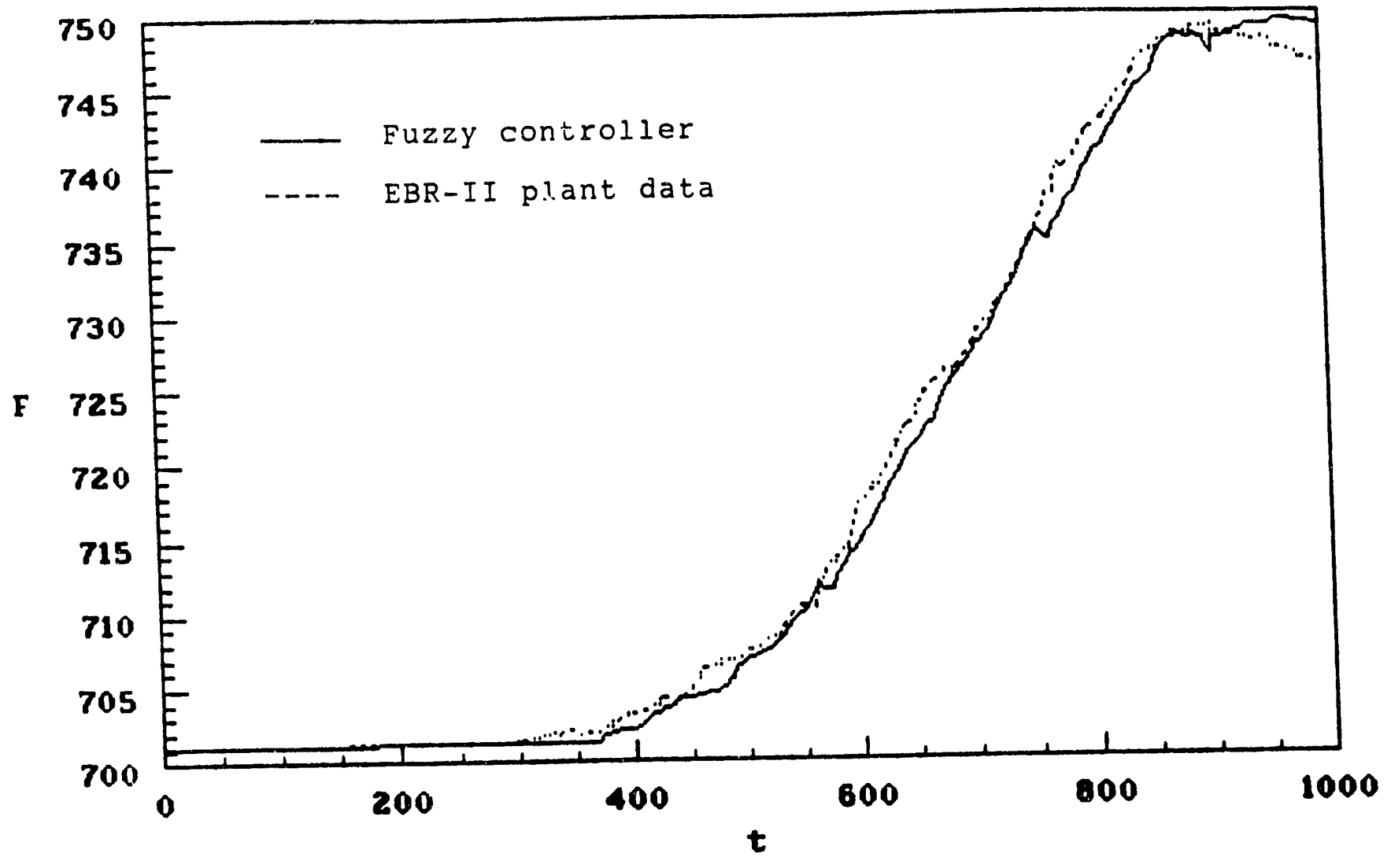

Figure 4 . Comparison of reactor core-exit coolant
temperature (F) due to fuzzy controlier and the EBR-II plant data. 


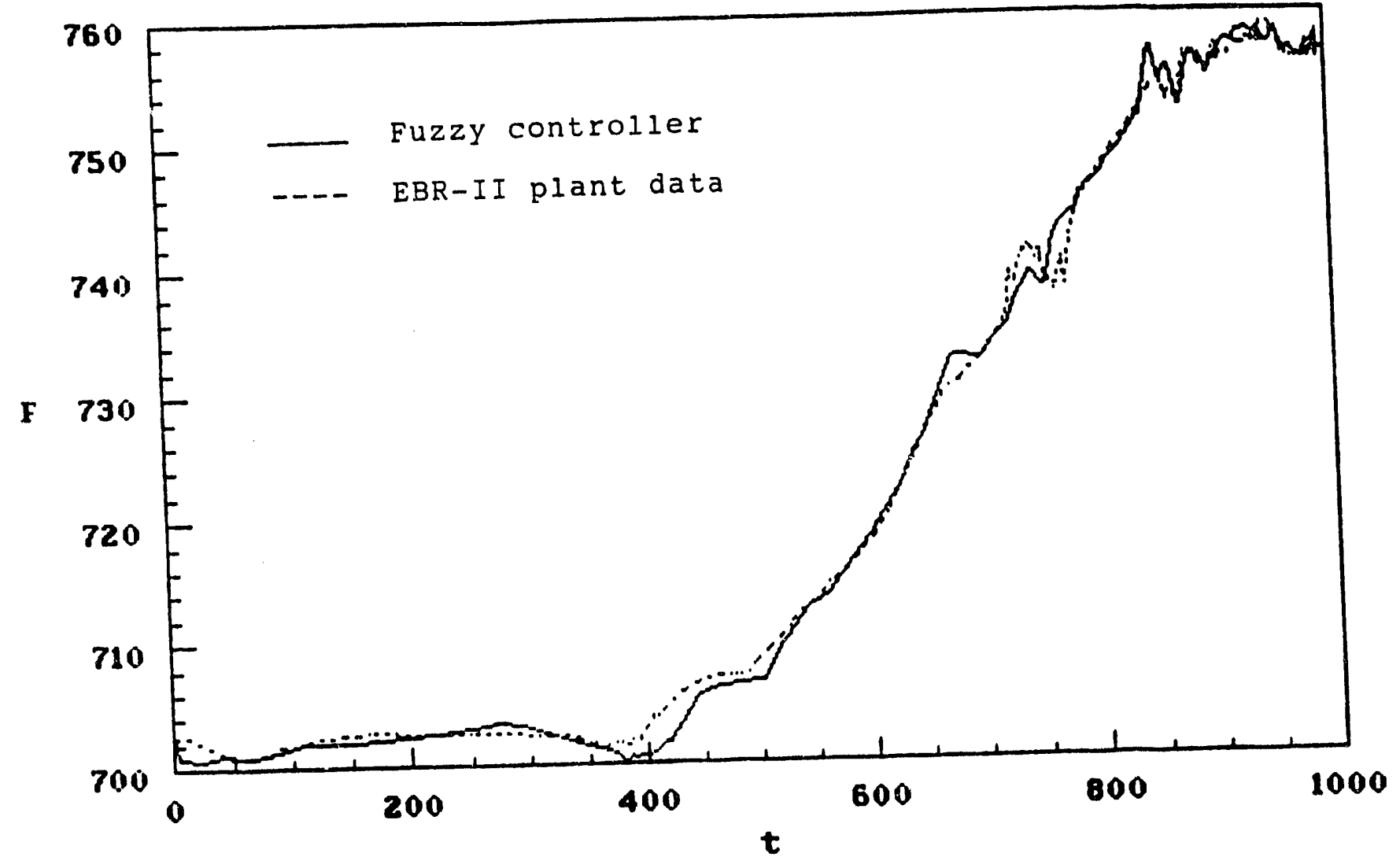

Figure 5. Comparison of IHX secondary sodium outlet
temperature (F) response to fuzzy controlier
and the EBR-II plant data. 


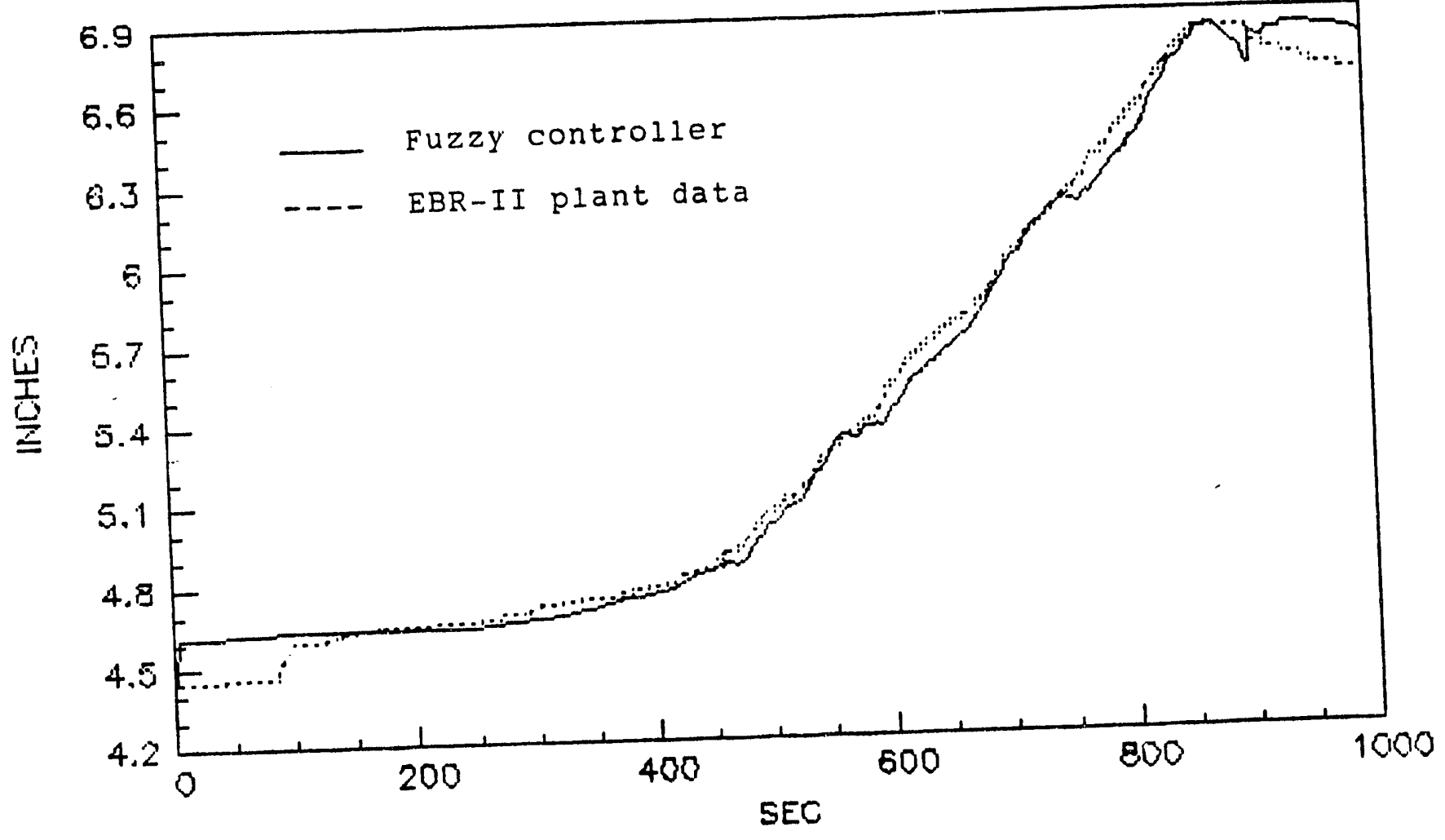

Figure 6. Comparison of control rod bank 11 position (inch) as designed by the fuzzy controller and the actual EBR-II plant data. 


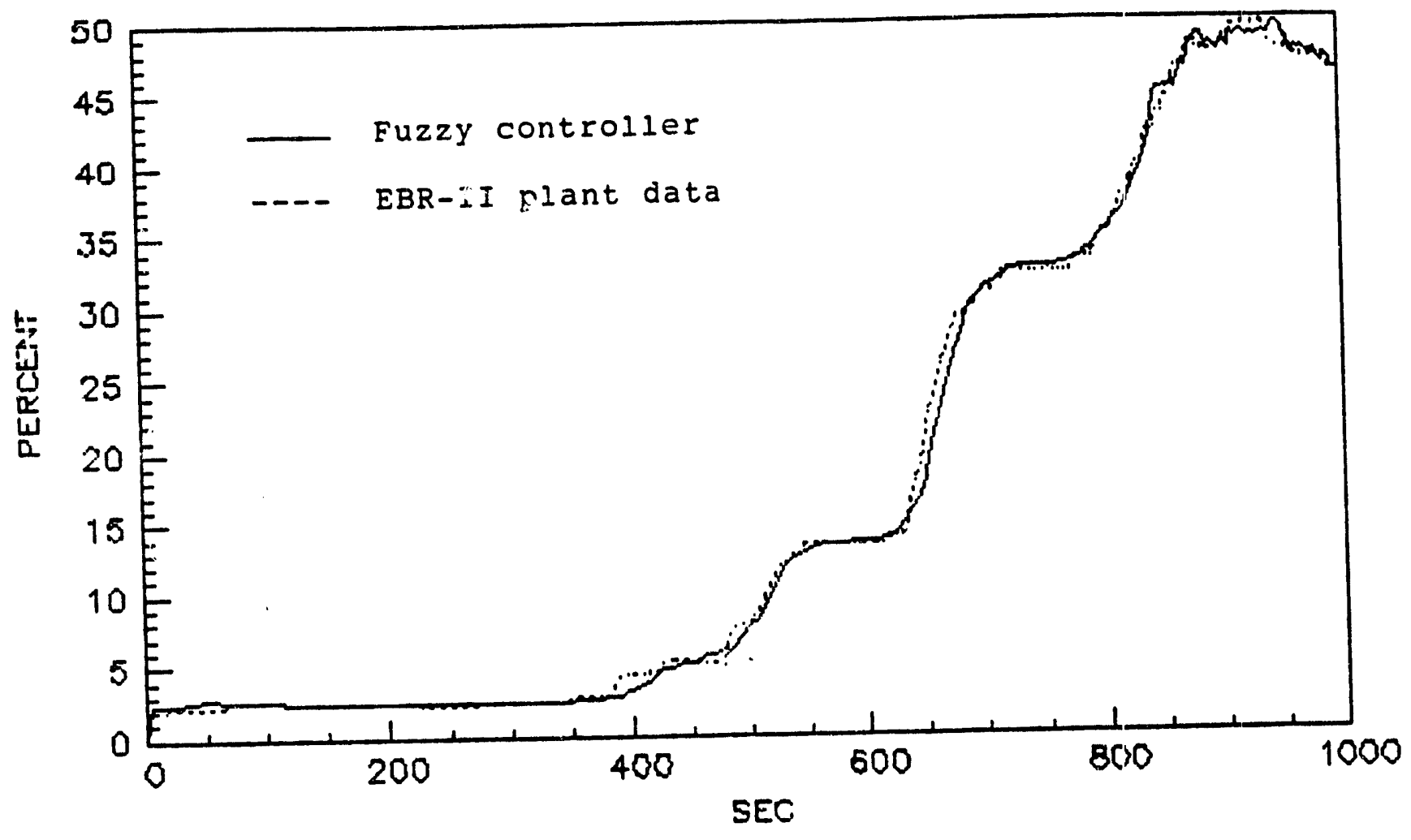
Figure 7 . Comparison of secondary sodium flow rate ( $\%)$
as desogned by the fuzzy controller arid the actual EBR-II plant data. 

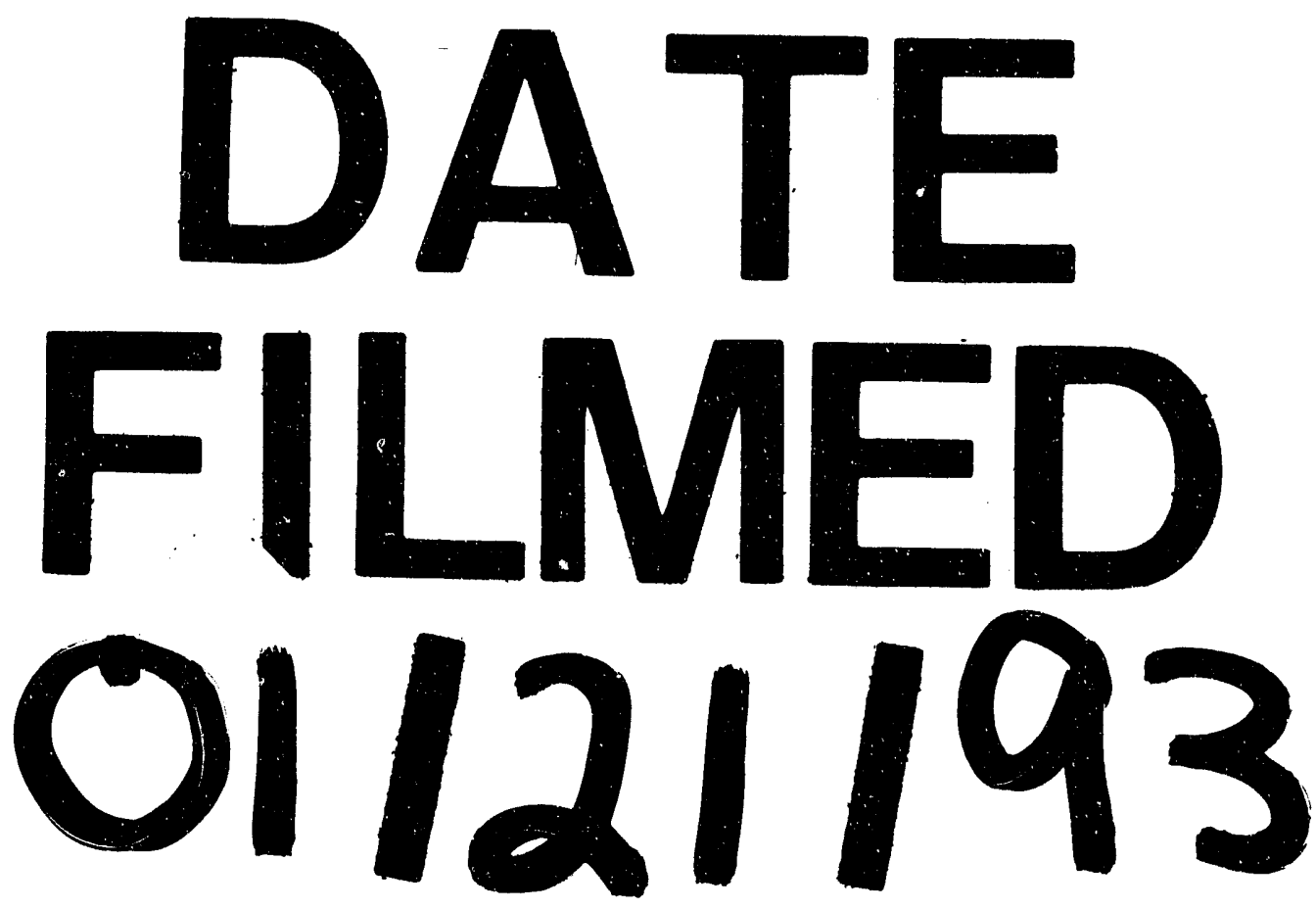
